\title{
Contribuições da antropologia médica para a medicina
}

\author{
Contributions of medical anthropology for medicine
}

\author{
Marcia Thereza Couto
}

Couto MT. Contribuições da antropologia médica para a medicina / Contributions of medical anthropology for medicine. Rev Med (São Paulo). 2012 jul.-set.;91(3):155-8.

\begin{abstract}
RESUMO: Trata-se de um artigo de revisão que aborda as contribuições da Antropologia Médica para o campo da educação médica. A discussão centra-se na reflexão de como as noções e comportamentos ligados aos processos de saúde e de doença integram a cultura de grupos sociais onde os mesmos ocorrem. Argumenta-se que os sistemas médicos de atenção à saúde, assim como as respostas dadas às doenças, são sistemas culturais, consonantes com os grupos e realidades sociais que os produzem. A compreensão dessa relação se mostra fundamental para a formação médica.
\end{abstract}

DESCRITORES: Antropologia médica/educação; Educação médica, Cultura.

A Antropologia reivindica a saúde e a doença como objetos próprios e fecundos de estudo, pois estes são fenômenos constitutivos da dimensão social da vida humana. Igualmente estuda a medicina, os doentes e os profissionais de saúde como parte de suas questões. Tal empenho fundamenta-se no reconhecimento de que o processo saúde-doença, embora geralmente assentado em um substrato biológico, ganha, pela cultura, sentidos e significados para o indivíduo e seu grupo de pertencimento e, igualmente, possibilidades de ação/intervenção sempre concernentes ao grupo social em questão. Nestes termos, saúde e doença são apreendidas pela Antropologia como expressão da relação entre fatores biológicos, sociais, políticos, econômicos, culturais e ambientais, conformando 'fenômenos sociais totais"1.

\begin{abstract}
This is a review article that discusses the contributions of medical anthropology to the field of medical education. The discussion focuses on reflection as to how notions and behavior related to the processes of health and illness are an integral part of the culture of the social group in which they occur. It is argued that medical and health care systems are cultural systems consonant with the groups and social realities that produce them. Such a comprehension is fundamental for the medical training.
\end{abstract}

KEYWORDS: Anthropology, medical; Education, medical; Culture.

As análises antropológicas dos processos de saúde-doença ganharam força a partir da década de 1950. Nas décadas seguintes estabeleceram-se subcampos disciplinares como a Antropologia Médica e Antropologia da Saúde e da Doença, originalmente nos EUA, com posterior e reconhecida expansão em países da Europa, como França e Inglaterra, no Canadá e alguns países da América Latina, em especial Brasil e México². Desde os anos 1980 assiste-se a uma produtiva colaboração entre Antropologia e Medicina, tanto em termos de pesquisas como na formulação e implantação de programas de saúde culturalmente informados. Desta produtiva cooperação, sobressaem os vários e conceituados periódicos que veiculam resultados de pesquisa, debates e proposições teórico-metodológicas e as associações acadêmicas da área (a título de exemplo

Cientista Social, Mestre em Antropologia, Doutora em Sociologia. Professora do Departamento de Medicina Preventiva, Faculdade de Medicina, Universidade de São Paulo.

Endereço para correspondência: Marcia Thereza Couto. Av. Dr. Arnaldo, 455, $2^{\circ}$ andar, sala 2177, Cerqueira César, São Paulo, SP. CEP: 01246-903. e-mail: marthet@usp.br 
destacam-se periódicos como Social Science \& Medicine, Medical Anthropology, Medical Anthropology Quarterly, Medical Anthropology: Cross-Cultural Studies in Health and IIIness, Anthropology \& Medicine). Ressalte-se, também que a seção de Antropologia Médica é uma das mais prestigiadas da Associação Norte-Americana de Antropologia). A expressão desta colaboração também pode ser vista no crescimento consistente de publicações em base de dados científicas. Em levantamento realizado na Biblioteca Virtual da Saúde (BVS) ${ }^{17}$, em 15/09/2012, com as expressões 'antropologia médica' e 'medicina', no campo 'assunto', foram localizados 1.379 publicações entre 1992 e 2011. Na década de 1990, tinha-se cerca de 33 artigos publicados por ano; a partir dos anos 2000 a média de artigos por ano sobe para 62 . As três principais revistas nas quais as pesquisas são publicadas são: Social Science \& Medicine, Medical Anthropology e Medical Anthropology Quarterly. A maior parte dos estudos apoia-se em metodologia qualitativa (destacando-se os estudos de caso, as etnografias e os relatos de caso), embora também mereçam destaque os estudos epidemiológicos (especialmente os de prevalência) em associação com abordagens qualitativas. A leitura dos resumos dos 181 artigos disponibilizados na íntegra nesta base de dados permite sugerir que o conceito de cultura e as análises fundamentadas a partir deste se apresentam como importantes referências das publicações. Neste sentido, é a partir deste conceito, basilar para a Antropologia, que se pretende apontar contribuições do conhecimento socioantropológico sobre o processo de saúde-doença para a Medicina, destacando-se temáticas, objetos e abordagens do debate acadêmico atual, bem como as potencialidades deste debate para a formação médica.

A Antropologia como disciplina científica se funda num certo paradoxo da natureza humana: a unicidade biológica associada à capacidade praticamente infinita para criar formas de organização social e construir símbolos e significados que dão sentindo aos modos de vida. Assim, o conceito de cultura pretendeu, desde a origem da Antropologia, fornecer as bases para o projeto de reconhecer e compreender uma humanidade plural ${ }^{3,4}$. A discussão sobre as várias formulações analítico-teóricas do conceito de cultura foge aos limites deste artigo. A conceitualização adotada a seguir visa potencializar a discussão sobre aspectos essenciais e instrumentais que serão utilizados na reflexão sobre a atualidade do conceito nos debates da antropologia médica, especialmente quando aplicado à formação médica.

Cultura pode ser definida, na acepção de Clifford Geertz ${ }^{5}$, importante expoente da Antropologia Interpretativa, que fundamenta grande parte da produção da Antropologia Médica Norte-Americana, como um "universo de símbolos e de significados que permite aos indivíduos de um grupo interpretar a experiência e guiar as ações". Esta definição de cultura como sistemas entrelaçados de símbolos interpretáveis dentro dos quais os acontecimentos sociais, os comportamentos e as instituições podem ser descritos de forma inteligível, possibilita entender a saúde (e, em complemento, a doença) como um sistema que é social e cultural na sua origem, estrutura, função e significado.

A partir desta definição de cultura, Arthur Kleinman, médico psiquiatra, antropólogo e professor dos departamentos de Antropologia Médica e Psiquiatria Transcultural em Harvard (USA), desenvolveu modelos, noções e abordagens úteis às investigações colaborativas entre Medicina e Antropologia e, de modo decisivo, ao desenvolvimento de competências culturais para o manejo clínico.

As noções de illness e disease, desenvolvidas pela Antropologia Médica nos anos de 1980 ainda hoje repercutem na produção acadêmica de interface entre Antropologia e Medicina, a despeito das releituras mais recentes destas noções. Illness e disease ressaltam o aspecto de que as produções de significado acerca da doença remetem a visões de mundo que, expressas pelo indivíduo singular, são conformadas pela cultura da qual ele toma parte. Com a distinção entre illness e disease é possível compreender e se posicionar frente às tensões produzidas no "encontro" entre profissionais da saúde e usuários. Illness (pertubação, sofrimento, enfermidade) é a forma como os indivíduos e os membros de sua rede social categorizam e atribuem sentido aos sintomas. Illness é a resposta subjetiva do indivíduo à situação de doença, uma resposta que engloba aspectos individuais, sociais e culturais à experiência de estar doente. Por outro lado, disease (doença) é a forma como a experiência da doença (illness) é reinterpretada pelos profissionais de saúde com base no modelo biomédico que orienta seu trabalho clínico. Uma das atribuições do médico seria, portanto, "traduzir" o discurso, os sinais e os sintomas do paciente para chegar ao diagnóstico da doença, ou seja, decodificar illness em disease, isto é, passar da doença do doente para a doença do médico, na elaboração diagnóstica ${ }^{6}$.

As contribuições da Antropologia Médica também avançam no sentido de explicitar que todas as ações relacionadas com o cuidado à saúde são interrelacionadas e organizadas com o objetivo de enfrentar a doença, a desordem e o sofrimento dela decorrente para o enfermo/doente e seu grupo de pertencimento. O modelo de Sistemas de Cuidado de Saúde $(\mathrm{SCS})^{7,8}$ considera que as atividades de 
cuidado à saúde são respostas sociais organizadas frente à doença ${ }^{8}$. Neste modelo, a ênfase é posta de modo decisivo na maneira como cada um de nós, pertencentes à determinada cultura ou sub-cultura, 'aprendemos a ficar doentes' ou, em outras palavras, pensamos e agimos diante de uma situação de doença, optando por um determinado tipo de tratamento e avaliando seu resultado ${ }^{9}$. Segundo Helman ${ }^{10}$, quanto maior e mais complexa a sociedade, maior a possibilidade e disponibilidade de alternativas terapêuticas. Este pluralismo terapêutico envolve a coexistência de muitas e diferentes possibilidades de cura/tratamento e a liberdade de opção para as diferentes pessoas, lembrando-se que tais tratamentos, geralmente, têm premissas distintas (por exemplo: a medicina baseada nas ciências biomédicas, a mais utilizada em nosso meio; a medicina chinesa, mais conhecida pelo uso da acupuntura, uma de suas medidas terapêuticas; a medicina homeopática; entre outras). Para Kleinman ${ }^{5}$, nosso SCS é composto por três partes que por vezes se sobrepõem: o setor popular, o setor folk e o setor profissional. O primeiro (popular) é representado por pessoas próximas ao doente (amigos, familiares, vizinhos) e se constitui no espaço propriamente 'leigo' no qual a doença começa a ser definida e, a partir daí, são desencadeadas as várias possibilidades terapêuticas de cura. O segundo (folk ou tradicional), contempla especialistas de cura, porém sem regulamentação oficial, como benzedeiras, práticas religiosas e outras formas alternativas de cura. O terceiro (profissional) representa a prática hegemônica e formal de saúde que, na nossa sociedade, tem a Biomedicina como referência primordial, mas que também pode incluir a homeopatia e a acupuntura, por exemplo. A partir deste modelo, compreende-se que cada sub-sistema apresenta uma forma de explicar a doença e alternativas ou terapêuticas para enfrentá-la. Tal contribuição traz elementos para relativizar o papel da biomedicina entre os demais sub-sistemas de cuidado e, igualmente, reconhecer que, no nosso contexto, a relação entre os três subsistemas não implica, na maior parte dos casos, em oposições, concorrências e antagonismos entre si, mas complementaridades.

Outro tema recorrente nas produções da Antropologia Médica e que merece destaque é a noção de experiência da enfermidade ${ }^{11,12}$. Emergida no início da década de 1980 a partir dos trabalhos de Byron Good e Arthur Kleinman, ambos professores da Universidade de Harvard, esta noção fundamentou pesquisas inicialmente voltadas para a interpretação de doenças crônicas. Mas, ao longo dos últimos anos, quase todas as áreas de interesse das ciências sociais em saúde têm explorado esta noção. De uma maneira geral, são trabalhos que expressam uma preocupação em compreender e problematizar como os indivíduos vivenciam uma "experiência de sentir-se mal". Partindo do pressuposto de que o adoecimento, a saúde e a morte não se reduzem a uma evidência orgânica, natural e objetiva, são enfatizados os modos pelos quais suas vivências ou experiências pelas pessoas e pelos grupos sociais estão intimamente relacionadas às características organizacionais e culturais de cada sociedade ou grupo social específico e, igualmente, referem-se ao mundo subjetivo dos indivíduos. Trata-se, pois, da experiência do adoecimento enquanto realidade subjetiva tanto quanto intersubjetiva. A dupla dimensão da experiência da enfermidade reforça, ainda, o entendimento da doença/enfermidade como mediador das relações sociais. Doença/enfermidade está no centro das relações sociais, sendo definida por e redefinindo posições sociais e as relações nos ambientes da família, trabalho, lazer, afetividade-sexualidade, entre tantos outros. Deste modo, o impacto da doença/enfermidade expande-se para muito além da relação médico-paciente-cuidador.

Disto decorre outra contribuição da Antropologia Médica acerca dos processos saúde-doençacuidado, que aqui chamaremos abordagem da relação médico-paciente (que pode ser estendida aos demais profissionais de saúde e seus clientes). Entre os diversos aspectos sob os quais se vem estudando essa complexa relação, destacaremos aqui a perspectiva comunicacional. A comunicação é entendida no sentido de que, para além do encontro físico entre profissional de saúde e paciente no contexto da assistência, estão colocadas em interação diferentes experiências, compreensões e expectativas acerca do problema a ser cuidado, isto é, diferentes produções de significados em cada um dos lados dessa relação ${ }^{13}$. Assim, a cultura de cada sujeito estará igualmente presente e interferindo neste 'encontro'. Em recentes trabalhos Kleinman e Benson ${ }^{14}$, apontam que a competência cultural do médico tem sido tema bastante debatido nos últimos anos, especialmente quanto à influência de fatores culturais no diagnóstico, na terapêutica e no cuidado. Entretanto, a potencialidade da competência cultural pode ser desacreditada quando a cultura passa a ser reduzida a uma habilidade técnica na qual os médicos devem ser treinados para desenvolver uma expertise ${ }^{15}$. Para os autores ${ }^{14}$, este problema decorre de como o conceito de cultura é geralmente usado em medicina, o que contrasta notavelmente com o seu uso no campo da antropologia. No campo médi$\mathrm{co}$, Cultura é tida frequentemente como sinônimo de etnia, nacionalidade e língua. Por exemplo, pacientes de uma determinada etnia, tais como, o "bolivianos", "coreanos" etc., são considerados como possuindo 
um conjunto de crenças sobre a doença devido a traços étnicos tomados de maneira fixa. Seguindo-se este parâmetro, a competência cultural torna-se um mero modo de operar a conduta clínica em termos de "fazer" e "não fazer", apoiado num reconhecimento padronizado da cultura étnica do paciente.

Como buscamos demonstrar a partir da breve revisão da produção da Antropologia Médica, a antropologia concebe que os processos culturais que envolvem saúde-adoecimento-cuidado são complexos e inseparáveis de fatores econômicos, políticos, religiosos, psicológicos e de condições biológicas. As noções e os modelos anteriormente discutidos (IIIness, disease, experiência da enfermidade, sistemas de cuidado em saúde), que atualmente são empregados nos currículos de escolas médicas americanas ${ }^{14,16}$, devem ser tomados não como uma mera técnica de entrevista que tenta entender o paciente e o seu mundo social em termos do quanto ele afeta e é afetado pela doença/enfermidade. Ao

\section{REFERÊNCIAS}

1. Couto MT, Schraiber LB, Ayres JRCM. Aspectos sociais e culturais da saúde e da doença. In: Martins M, Carrilho FJ, Castilho EA, Alves VAF, Cerro GG, organizadores. Tratado de clínica médica. São Paulo: Manole; 2009. v. 1, p.350-6.

2. Nunes ED. Sociologia da saúde: história e temas. In: Campos GW, et al., organizadores. Tratado de saúde coletiva. São Paulo: Hucitec/Fiocruz; 2006. p.9-52.

3. Laplantine F. Aprender antropologia. São Paulo: Brasiliense; 2002.

4. Langdon EJ, Wiik FB. Antropologia, saúde e doença: uma introdução ao conceito de cultura aplicado às ciências da saúde. Rev Latinoam Enferm. 2010;18(3):17481.

5. Geertz C. A interpretação das culturas. Rio de Janeiro: LTC ed.; 1989.

6. Kleinman A. Illness narratives suffering, healing, and the human condition. New York: Basic Books; 1989.

7. Rhodes LA. Studying biomedicine as a cultural system: In: Sargent C, Johnson T. Medical anthropology: contemporary theory and method. Westport: Praeger; 1996. p.165-80.

8. Kleinman A, Eisenberg L, Good B. Culture, illness, and care: clinical lessons from anthropologic and crosscultural research. Focus. 2006;4(1):140-9.

9. Oliveira FA. Antropologia nos serviços de saúde: integralidade, cultura e comunicação. Interface Comunic contrário, estas noções devem ser consideradas no contexto clínico de modo a promover o diálogo a partir de uma escuta qualificada pelo médico acerca do significado e da experiência da doença do paciente. Nas palavras de Kleinman e Benson" "The moment when the human experience of illness is recast into technical disease categories something crucial to the experience is lost because it was not validated as an appropriate clinical concern" (p. 294).

Assim, ressaltamos a potencialidade destes aportes da Antropologia Médica para a formação em medicina e para o manejo da atividade clínica. Afirmamos que 'encontro' entre o profissional de saúde e o paciente/usuário remete a uma relação efetiva entre sujeitos que partilham de universos culturais próprios e que a 'comunicação' resultante do 'encontro' trará benefícios para ambos, pacientes e profissionais, no sentido do resgate da humanização do cuidado e da integralidade da atenção à saúde.

Saúde Educ. 2002;6(10):63-74.

10. Helman CG. Cultura, saúde e doença. Porto Alegre: Artes Médicas; 1994.

11. Alves PC. A experiência da enfermidade: considerações teóricas. Cad Saúde Publ. 1993;9(3):263-71.

12. Alves PC. A fenomenologia e as abordagens sistêmicas nos estudos sócio-antropológicos da doença: breve revisão crítica. Cad Saúde Pública. 2006;22(8):1547-54.

13. Ayres JRCM. Cuidado e reconstrução das práticas de saúde. In: Minayo MCS, Coimbra Jr CE, organizadores. Críticas e atuantes: ciências sociais e humanas na América Latina. Rio de Janeiro: Ed. FIOCRUZ; 2005. p.91-108.

14. Kleinman A, Benson P. Anthropology in the clinic: the problem of cultural competency and how to fix it. PLoS Med. 2006;3(10):e294.

15. DelVecchio GM. American medicine: the quest for competence. Berkeley: University of California Press; 1995.

16. Smith CS, Magdalena M, Hill W, Francovich C, McMullin J, Chavez L, Rhoads C. Cultural consensus analysis as a tool for clinic improvements. J Gen Intern Med. 2004;19:514-8.

17. Biblioteca Virtual da Saúde (BVS). Disponível em: http://pesquisa.bvsalud.org/regional/index.php 